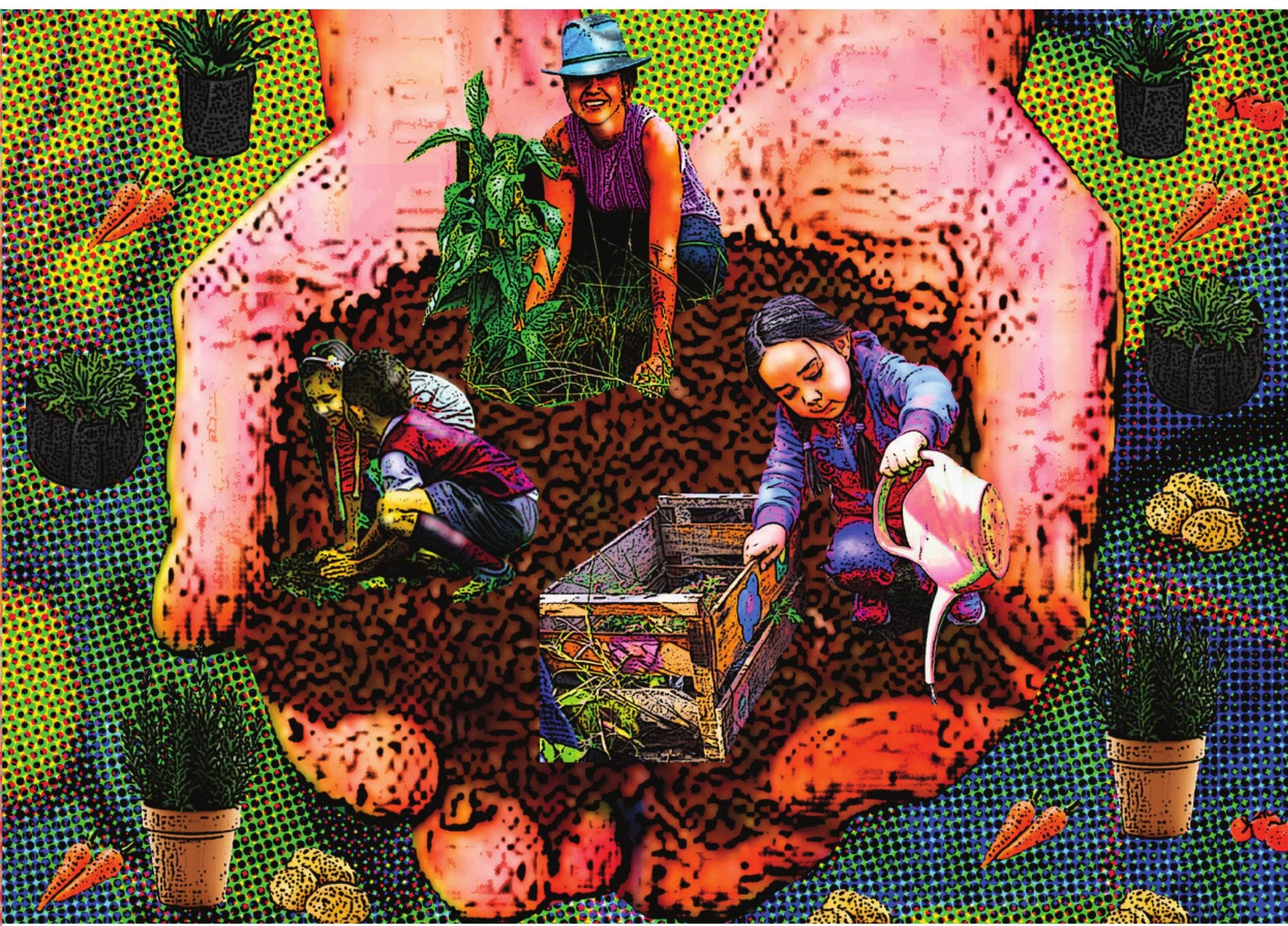

\title{
Nichos agroecológicos. El nicho como laboratorio de aprendizaje
} AGROECOLOGICAL NICHES; THE NICHE AS A LEARNING LABORATORY

NICHOS AGROECOLÓGICOS; O NICHO COMO LABORATÓRIO DE APRENDIZAGEM

Yenny Esperanza Parra Cortés

Ruby Marcela Reyes Aguirre

Edwin Oswaldo Villalba Vargas 
TEMA: : IMPORTANCIA DE LA INVESTIGACIÓN DE LOS MAESTROS Y MAESTRAS

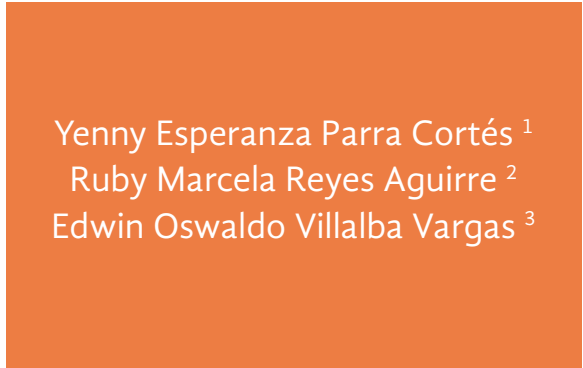

Licenciada en química, Universidad Pedagógica Nacional; Especialista en Docencia de las Ciencias para el Nivel Básico, Universidad Pedagógica Nacional; Magíster en Docencia de las Ciencias Naturales, Universidad Pedagógica Nacional; Directivo docente, Coordinadora SED, Colegio Gran Yomasa, IED; correo electrónico: yenyhope@hotmail.com

2. Licenciada en Educación Especial, Universidad Pedagógica Nacional; Magister en Escritura, Universidad Nacional de Colombia; Docente de básica primaria, Colegio Almirante Padilla, IED; correo electrónico: educmarce@hotmail.com

3. Licenciado en Biología, Universidad Distrital; Especialista en Docencia de las Ciencias para el Nivel Básico, Universidad Pedagógica Nacional; Magíster en Docencia de las Ciencias Naturales, Universidad Pedagógica Nacional; Directivo docente, Coordinador SED, Colegio Entre Nubes Sur-oriental, IED; correo electrónico: edwinvillalba@hotmail.com

DOI https://doi.org/10.36737/01230425.n38.2020.2318

Fecha de recepción: 3 de febrero de 2020 / Fecha de aprobación: 15 de mayo de 2020

\section{Resumen}

El presente artículo expone la historia de la experiencia pedagógica: "Nichos agroecológicos: una propuesta para formar líderes ambientales"; llevada a cabo en el Colegio Almirante Padilla, ubicado en la localidad de Usme. La propuesta surge de la necesidad de responder a las problemáticas ambientales enfrentadas por la localidad y la institución, así como del afán por fomentar la capacidad de gusto y disfrute por las actividades ecológicas. El proyecto busca formar a los estudiantes como líderes investigadores y transformadores de su entorno; actualmente se ha fortalecido con la conformación de un semillero investigativo.

Palabras clave: Semillero, nicho, investigación, líder, ambiente, agroecología.

\section{Abstract}

This text shows the history of the pedagogic experience "Agroecological niches: a proposal to form environmental leaders" from the Almirante Padilla school from the Usme borough in Bogotá, Colombia. This proposal arises from the necessity of answering the environmental issues that both the borough and the school are facing, as well as the liking and enjoyment for the activities that show an ecological character. The project seeks to educate the students as investigative leaders and transformers of their surroundings. Currently, the experience has been strengthened by the conformation of an investigational group.

Keywords: Investigational group, niche, investigation, leader, environment, agroecology.

\section{Resumo}

Este texto apresenta a história da experiência pedagógica "Nichos Agroecológicos: uma proposta para formar líderes ambientais", da escola Almirante Padilla, localizada no bairro de Usme em Bogota, Colômbia. Esta proposta surge da necessidade de responder aos problemas ambientais enfrentados pela cidade e pela instituição, bem como do gosto e da fruição de atividades de carácter ecológico. O projeto procura formar estudantes como líderes de pesquisa e transformadores de seu ambiente. Atualmente a experiência tem sido reforçada com a formação de um grupo de pesquisa.

Palavras-chave: Grupo de pesquisa, nicho, pesquisa, líder, ambiente, agroecologia. 
“Je est un autre"

(Yo soy otro).

Rimbaud, 1871.

S e ha elegido esta frase de Rimbaud para presentar el presente artículo porque, sin duda, la vida del maestro cambia cuando se investiga desde la escuela: se es otro. Así se transformaron nuestras vidas con la experiencia educativa "Nichos agroecológicos: una propuesta para formar líderes ambientales", que surgió hacia el año 2015 en la Institución Educativa Distrital (IED) Almirante Padilla, con el nombre de "Animales para aprender".

La experiencia continúa en la actualidad, más fuerte y organizada, logrando conformar un semillero investigativo. El proyecto nació como respuesta a las problemáticas enfrentadas por la institución y la localidad; factores como la acumulación y el manejo inadecuado de residuos sólidos en los alrededores de la escuela, el deterioro del suelo, la extinción de los recursos naturales, la acumulación de basuras en zonas baldías, el aumento de fauna callejera y la contaminación de las quebradas Yomasa y Bolonia; todo ello generó sentimientos de desarraigo en los habitantes de la localidad (Usme) y vecinos de la institución, quienes llegaron a desconocer el valor de su territorio.

Para atender dichas problemáticas se buscó empoderar estudiantes que se caracterizaran por su liderazgo y capacidad para replicar aprendizajes, quienes tenían como misión principal generar hipótesis para atender las prácticas que nutrían las problemáticas ambientales en la institución. La dinámica llevó a que los estudiantes tomaran la iniciativa de crear sub-proyectos con un enfoque investigativo, de acuerdo con cada una de las problemáticas detectadas; así nacieron los nichos o grupos de trabajo ${ }^{4}$.

Entonces, la propuesta del proyecto es formar estudiantes líderes, dinamizadores e investigadores, con conciencia ambiental, capaces de reconocer, formular y aplicar acciones educativas a través de la puesta en marcha del proyecto ambiental escolar PRAE. De igual manera, se busca relacionar los principios y acciones propias del proyecto con lo expresado en el modelo pedagógico de la institución (crítico-social), logrando que la investigación permee

4. El nicho describe el estatus de un animal en su comunidad, para indicar lo que está haciendo y no solo lo que parece (Elton, 1927). las prácticas pedagógicas mediante el proyecto, en el sentido planteado por Reyes.

[...] la investigación como una propuesta transformadora de saberes en la escuela y al servicio de la comunidad hace que las asignaturas cobren sentido en el espacio real y común. En esta medida, investigar se asocia a la resolución de problemas: es usar el conocimiento para nuestro provecho (Reyes, 2019, p. 135).

Investigar desde la práctica pedagógica implica indagar, lo cual nos ha significado trazar un camino guiado por conjeturas que, a veces, llevan a regresar y reflexionar sobre la manera de abordar las "hipótesis". El trabajo se vincula con el análisis y apropiación de problemáticas propias de nuestro contexto, haciéndonos parte de ellas, por eso ha implicado conocernos y acercarnos a las distintas situaciones desde el interés por construir aquello que en algún momento llamamos "conocimiento", pero desprovisto del estatus de poder que ha jerarquizado la escuela en algunas realidades. Sin embargo, el elemento más destacable es que todo involucra el disfrute por aprender, por el proceso creador y constructivo que siempre antecede a lo creado, a lo transformado.

El camino recorrido quizás no se ajuste al trazado por la rigurosidad de la investigación como metodología, pero sí ha representado una ruta para explorar y proponer soluciones en nuestro contexto inmediato. El semillero de investigación se ha convertido en espacio de encuentro para actores con intereses comunes: estudiantes, maestros, vecinos, facilitadores. Al aproximarnos a una posible actividad investigativa se pone a prueba el método empírico analítico, que ha servido como itinerario en la búsqueda de respuestas. La dinámica de los nichos como semilleros de formación de líderes ambientales se desarrolla, inicialmente, desde la invitación a estudiantes que, por iniciativa propia, quieran formar parte de ellos.

Con la detección de problemáticas ambientales, y una lluvia de ideas para determinar la creación de un nicho que dará origen a un sub-proyecto, los estudiantes se agrupan en dichos sub-proyectos o nichos de acuerdo con sus intereses y plantean objetivos, posibles acciones e hipótesis frente al plan de trabajo. Durante la ejecución de los sub-proyectos deben informar constantemente al grupo general y dar cuenta de sus avances.

\section{Del cómo empezamos: Animales para aprender}

El conocimiento científico se ha construido mediado por características históricas, sociales, políticas e ideológicas, dando origen a teorías, leyes y datos, informaciones que tienden a 
TEMA: : IMPORTANCIA DE LA INVESTIGACIÓN DE LOS MAESTROS Y MAESTRAS

entenderse como únicas, verdaderas e inmutables, asumiendo que deben aprenderse, recitarse y aplicarse tal cual, desconociendo el contexto histórico, cultural y social. Para llegar a comprender diferentes hechos y fenómenos del mundo natural, y las relaciones que se generan en él, se considera esencial construir explicaciones en el proceso de enseñanza-aprendizaje. La experiencia aquí narrada se enmarca en diversos cuestionamientos desde la reflexión crítica sobre el quehacer del maestro de ciencias en el aula de clase.

Nuestra práctica pedagógica es construida y puesta en escena en el Colegio Almirante Padilla IED, lugar donde confluye nuestro saber y se convierte en centro de aprendizaje. Al interior de la institución, el área de ciencias naturales y educación ambiental lidera el Proyecto Ambiental Escolar -PRAE: "Educación ambiental en el Colegio Almirante Padilla: proceso para el desarrollo sostenible"; el cual se orienta hacia la puesta en marcha de seis líneas de acción, propuestas en los lineamientos del Plan Institucional de Gestión Ambiental (PIGA) de la SED, y una adicional, planteada por el área, denominada "Animales para aprender", que se incorpora al PRAE desde el año 2008.

La finalidad del trabajo era fortalecer el interés por la ciencia y generar procesos de acercamiento de los estudiantes a las especies animales, para inculcar el respeto y cuidado por ellas. Durante 2010 nació la experiencia "Acuarios para aprender" y se adquirieron acuarios y peces con el apoyo del programa ONDAS (estrategia fundamental de Colciencias para el fomento de una cultura ciudadana y democrática en la Población infantil y juvenil colombiana); sin embargo, se trataba de prácticas aisladas de un problema de conocimiento.

Dada la importancia de investigar e innovar en la clase de ciencias, en 2013 los acuarios brindaron la posibilidad de reconocerles como un escenario para que los estudiantes construyan explicaciones. Para 2014, se plantea el estudio de las condiciones de vida de los peces de acuario desde un ámbito social, político, económico y cultural que contribuye a la constitución de los estudiantes como sujetos sociales. Igualmente, para este año se adquirió una dotación de acuarios $^{5}$, gracias a las INCITAR (Iniciativas Ciudadanas de Transformación de Realidades).

En 2015 se integró el eje tecnológico, consolidando la experiencia "Dinámicas de peces de acuario como posibilidad de construcción de conocimiento en el aula"; proceso que se realiza con el

\footnotetext{
Ubicados en el patio central, las dependencias administrativas, la portería, la sala de maestros, las oficinas de directivos docentes y orientadores, y en las tres sedes de la institución.
}

acompañamiento de C4 (Convenio entre la Secretaría de Educación del Distrito y el Centro Ático de la Pontificia Universidad Javeriana), donde los estudiantes potenciaron el trabajo colectivo y creativo en ciencia y tecnología, fortaleciendo el uso e implementación de las TICS en su experiencia; como prueba de ello es posible consultar la página: http://mariiapaula2312.wix.com/aqua, de autoría estudiantil.

Todo el trabajo ha contado con reconocimientos, a través de expediciones pedagógicas lideradas por la DILE de la localidad; con ellas los estudiantes han tenido la posibilidad de viajar a la Isla Gorgona y al Valle del Cauca; igualmente, se han dado expediciones pedagógicas lideradas por la SED, en la modalidad de Bogotá Región, que dieron la oportunidad de visitar los Llanos Orientales.

\section{Del cómo vamos: Creación de un nicho}

El proyecto de "Nichos Agroecológicos: Una propuesta para formar líderes ambientales", surge desde el reconocimiento de las problemáticas enfrentadas por nuestra institución y la localidad, que dieron la oportunidad de buscar formas de empoderar estudiantes con rasgos de liderazgo y que se destacaran como agentes replicadores de aprendizajes; tenían como principal misión generar hipótesis frente a las prácticas realizadas que involucraban problemáticas ambientales del colegio.

A partir de esta dinámica, los estudiantes tomaron la iniciativa de crear sub-proyectos con un enfoque investigativo, de acuerdo a cada una de las problemáticas detectadas. Inicialmente se unieron al proceso de manera voluntaria, postulando dos estudiantes por curso como formadores y líderes ambientales; posteriormente, recibieron diferentes capacitaciones que involucraron saberes relacionados con las características propias de un investigador y líder, a la vez que las responsabilidades que se adquieren frente al proceso.

De la misma manera, se realizó un recuento del proyecto de formación y se discutieron las problemáticas ambientales que serían susceptibles de ser intervenidas y de facilitar la generación de hipótesis. Ubicados en cada uno de los nichos, escogidos por ellos voluntariamente, se realizaron capacitaciones por grupos de trabajo, incorporando temas como: Qué es una investigación, cómo se desarrolla un proceso investigativo y cuál es la ruta a seguir en la investigación de ese nicho o sub-proyecto.

Posteriormente, se establecieron planes acción por cada nicho y se plantearon capacitaciones en función de cada uno de ellos, las formaciones se realizaron de manera general o por grupos con las entidades distritales que proporcionan apoyo. Al interior de cada 
educación

Ciudad

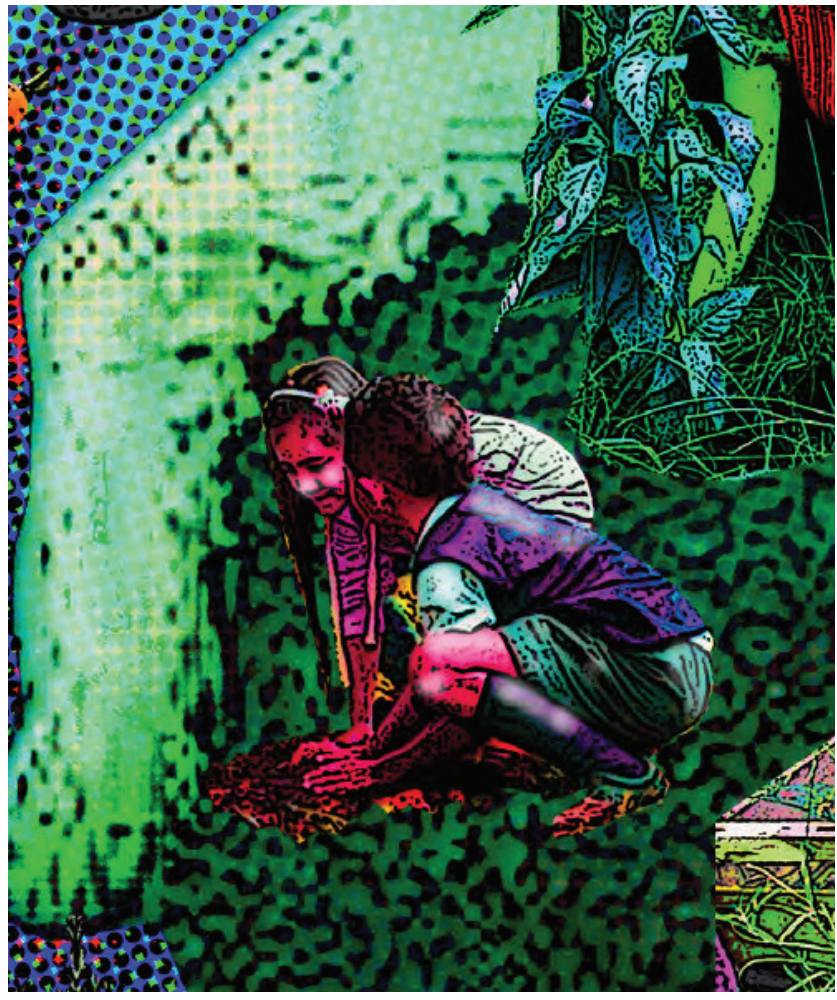

nicho se realiza un trabajo documental, estilo diario de campo, que se apoya en las clases de ciencias naturales y que, de acuerdo a los cursos, se une con el currículo a desarrollar.

El plan de acción se traza de acuerdo a la documentación creada y, luego, se procede a intervenir de acuerdo con la problemática detectada en cada nicho; en este momento, se busca dar respuesta a las problemáticas y comprobar las hipótesis formuladas frente ellas. Finalmente, se replica la información en los cursos y se evalúa el procedimiento aplicado, teniendo en cuenta las variables y realizando en clase un análisis estadístico de lo invertido y lo producido; dependiendo del trabajo realizado se emprende un análisis cualitativo.

Es importante señalar que los procesos de documentación y registro escrito son realizados en las asignaturas, para cada curso de ciencias naturales, física y química. El trabajo se ejecuta desde los grados quinto a 11, teniendo en cuenta el nivel de complejidad, porque cada vez que se desarrolla propiciamos la resignificación de la práctica docente, buscando guiar a los estudiantes hacia la posibilidad de dar significado y de reflexionar acerca de las relaciones entre el mundo, la ciencia y la sociedad; solo de esta manera se es otro.

Como ya se ha señalado, el proyecto nace al reconocer los problemas de la institución y la localidad, por lo que objetivo de empoderar a estudiantes con características de liderazgo y de interés por la investigación, se dirigió a permitirles la posibilidad de reconocer, diseñar, y aplicar acciones educativas a través de la puesta en marcha del proyecto ambiental escolar PRAE. Una vez los estudiantes han escogido voluntariamente uno de los nichos, se realizan capacitaciones por grupos de trabajo y se establecen planes de acción por cada nicho, adecuando la capacitación al mismo, siguiendo la ruta presentada anteriormente.

La metodología permitió que en 2017 comenzara la formación de líderes ambientales (dos representantes por curso), ampliando las líneas de acción a las formuladas en el proyecto de gestión de la huerta escolar, que contó con el apoyo y capacitación agrícola del Jardín Botánico de Bogotá. En este sub-proyecto se buscó investigar los cultivos urbanos que podrían implementarse en la huerta escolar, así como el estudio de suelos que se podría llevar a cabo durante el proceso de mejoramiento para cultivos.

Esta línea de investigación continúa siendo una de las insignias del proyecto; el Jardín Botánico brindó su apoyo para el manejo de cultivos de plantas medicinales, ornamentales, condimentarias y hortalizas, también para los procesos de lombricultura, biopreparados y compost, y para el reconocimiento, marcaje y documentación de individuos arbóreos de la huerta escolar, con lo que fue posible iniciar la documentación e implementación de cultivos casi extintos. Todo lo anterior cuenta con una base de consulta, documentación, hipótesis, efectos y variables.

Para el año 2018 se incorporó el manejo responsable del agua como un sub-proyecto de acción, para lo cual se planteó utilizar tanques recolectores de agua lluvia en la huerta escolar; de la misma forma, se propuso identificar y realizar obras de impacto en las fuentes hídricas cercanas al colegio (quebradas Yomasa y Bolonia) y en los cuerpos hídricos de la localidad. En 2019 se implementó el acompañamiento de la Secretaría de Ambiente y del Jardín Botánico de Bogotá, en materia de consulta y acciones para concretar recorridos en el territorio; las entidades han certificado a los estudiantes líderes ambientales en aspectos como: manejo de recursos sólidos, agricultura urbana y reconocimiento del territorio del Distrito Capital. En la actualidad, el proyecto está organizado a partir de cinco nichos: 
TEMA: : IMPORTANCIA DE LA INVESTIGACIÓN DE LOS MAESTROS Y MAESTRAS

1. Nicho de lombricultivo y huéspedes invertebrados. Subproyecto que surge como complemento de la huerta escolar y se planea desde el reconocimiento de especies invertebradas que ayudan al sostenimiento del suelo en la huerta y a la sostenibilidad del ecosistema; cuenta con un proceso de seguimiento de su impacto en la institución; la línea es la continuación del proyecto inicial "Animales para aprender". Al tiempo, aún se adelanta, junto a distintos responsables de la comunidad, la instalación de acuarios en algunos lugares del colegio; también se han identificado especies de invertebrados y polinizadores en la huerta, documentando su presencia e impacto.

2. Nicho de Banco de semillas e invernadero. La huerta es el espacio recuperado para el aprovechamiento de cultivos y de investigación en agrología y estudios básicos de suelos. El sub-proyecto se desarrolla con el acompañamiento del Jardín Botánico de Bogotá (Proyecto de agricultura urbana); en el nicho se reconoce y optimiza el proceso de la fotosíntesis con el uso de técnicas agrícolas, y se realizan procesos de intercambio de semillas y plántulas con otras instituciones. La ejecución de los trabajos se vio beneficiada con recursos físicos obtenidos gracias a la convocatoria de Semilleros escolares de investigación ${ }^{6}$.

3. Nicho o sub-proyecto documental de arbolado. Surge desde la iniciativa de reconocer los árboles presentes el colegio y su función en nuestro ecosistema; para concretar las labores se ha realizado un marcaje, un inventario documentado y se han creado cartillas didácticas para la comunidad educativa.

4. Nicho o sub-proyecto de manejo de residuos y posconsumo. Busca aplicar y desarrollar diferentes estrategias pedagógicas con la comunidad educativa y la localidad, para generar conciencia ecológica, espacios limpios y agradables, evitar los impactos negativos de los residuos en el medio ambiente y mejorar la calidad de vida de quienes se relacionan con nuestra institución. Para garantizar el éxito de las prácticas, los líderes ambientales realizan capacitaciones frecuentes sobre el manejo, separación y almacenamiento adecuado de residuos con los diferentes estamentos del colegio (servicios generales, administrativos, cafetería, vigilancia, estudiantes, docentes y vecinos).

IDEP (Instituto para la Investigación Educativa y el Desarrollo Pedagógico). Segunda convocatoria para el apoyo a la consolidación de semilleros escolares de investigación de docentes y directivos docentes del Distrito Capital, 2019.
5. La Huerta. Junto con el nicho del invernadero constituye la línea de biodiversidad en nuestro PRAE; se ha consolidado como laboratorio de aprendizaje para la recuperación de productos agrícolas considerados exánimes, y para la elaboración de compostaje.

\section{Un nicho como innovador de experiencias}

El nicho, como espacio de interacción, ha permitido la desaparición de la línea vertical de relación entre docente y estudiante; al tiempo, da valor a las diferentes miradas de aquello que reconocemos como "saber" y, sin duda, posibilita el desalojo de la figura de poder absoluto que muchas veces asumimos como docentes; en su lugar, permite pasar al lugar del aprendizaje en un sentido horizontal con el estudiante, mientras fortalece las relaciones interpersonales y de camaradería, pues los alumnos se congregan vinculados por un interés y logran reconocerse como líderes en un proceso.

El nicho, como posibilitador de saber, despierta el interés por aprender, por construir un conocimiento que va más allá del aula, que busca respuestas en el contexto, en el interior de cada

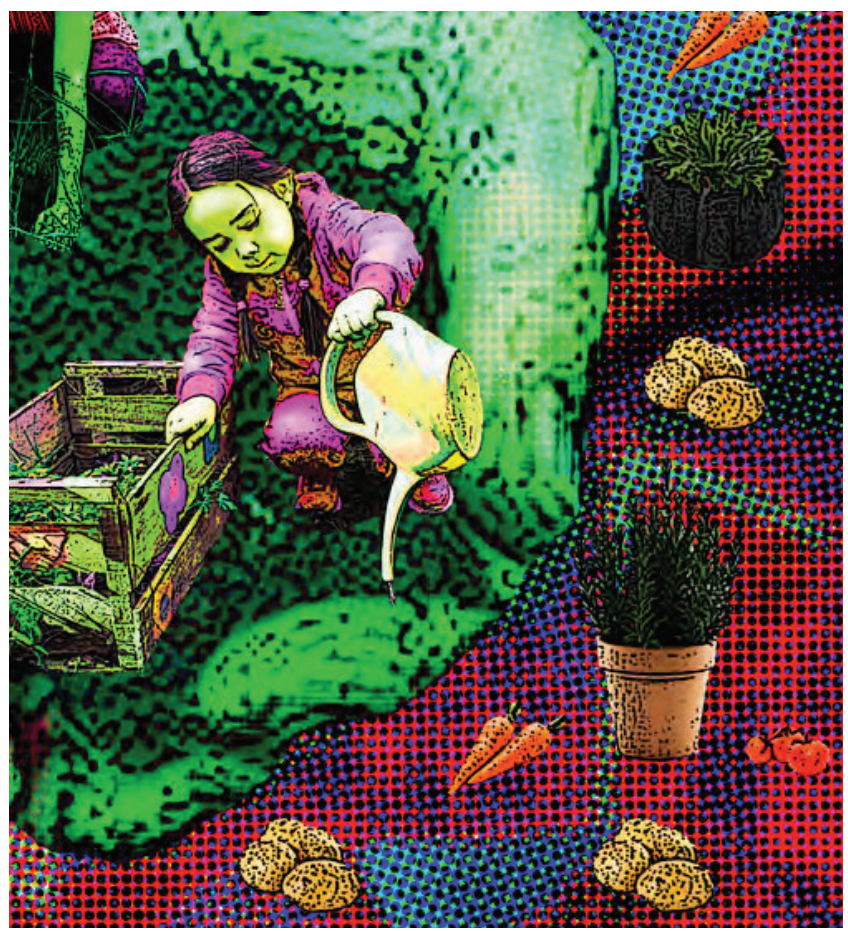


estudiante y en el accionar del grupo; ha sido, sin duda, la excusa para romper un modelo tradicional de acumulación de información, de segmentación de temas y asignaturas, y de jerarquización de saberes; resultando en un proceso que pone el conocimiento a nuestro servicio.

Desde su papel como formador de líderes, el nicho ha representado una forma de descubrir las fortalezas que, como docentes, tenemos en tanto facilitadores de aprendizaje, llevándonos a reconocer las capacidades de nuestros estudiantes, de tal manera que nos sea posible aprovecharlas al máximo en cada uno de los grupos de aprendizaje. Al tiempo, desde su lugar como laboratorio de conocimiento, el nicho es un agente que nos ha permitido ver cómo se construye, con una arista, entender el conocimiento como proceso y no como fin, lo cual permite que cada paso en el camino sea revelador y cobre sentido en el aula, en la casa, en la localidad. Lo hace significativo para la vida, recordando que:

La teoría del aprendizaje significativo es la propuesta que hizo David P. Ausubel en 1963 en un contexto en el que, ante el conductismo imperante, se planteó como alternativa un modelo de enseñanza/ aprendizaje basado en el descubrimiento, que privilegiaba el activismo y postulaba que se aprende aquello que se descubre. Ausubel entiende que el mecanismo humano de aprendizaje por excelencia para aumentar y preservar los conocimientos es el aprendizaje receptivo significativo, tanto en el aula como en la vida cotidiana Ausubel $(1976,2002)$. No es necesario, desde este enfoque, descubrirlo todo (Rodríguez, 2011, p. 30).

Si se parte de que los procesos se dan en forma evolutiva o de transformación, del proceso creador de aprendizaje hacia lo que se crea, es posible afirmar que un nicho es el lugar para investigar; existe allí una relación condicionante entre investigar y crear. Estas relaciones se establecen alrededor de algo que se quiere aprender y algo que se quiere crear, de una "motivación" o de un acto voluntario que conduce a un saber. Los nichos son, sin duda, asideros de experiencias significativas en un proceso de construcción de conocimiento, es saber que comienza a existir, que emerge y se materializa en respuestas, decisiones, inspiraciones y en semilleros de investigación.

En cuanto a la labor docente, es necesaria una formación holística, entendida en el sentido de un sistema complejo; los aspectos disciplinar, humanístico, pedagógico y didáctico se consolidan y conjugan en el maestro que busca trascender en su práctica, por ello, Vasco (1993) propone: "Reivindicar un concepto mucho más amplio de pedagogía, que incluya ante todo la reflexión, la explicitación, y la sistematización de la práctica y del saber pedagógico", entendido este proceso desde una re-significación del maestro, que aquí es capaz de propiciar y aportar en la construcción de una sociedad.

Al tiempo, es importante reconocer que, a pesar del esfuerzo de los maestros para que los estudiantes comprendan y asignen sentidos y significados a los fenómenos naturales, es posible encontrar ocasionalmente un sentimiento de insatisfacción que, en palabras de Pozo y Gómez (1990), se expresa como una: "creciente sensación de desasosiego, de frustración, al comprobar el limitado éxito de sus esfuerzos docentes".

En este orden de ideas, surge la necesidad de reflexionar sobre aspectos concernientes a la enseñanza de las ciencias en la escuela, entre ellos: las prácticas "transmisionistas"; la falta de contextualización de las temáticas abordadas en clase; y la escasez de espacios para construir explicaciones y conocimiento, especialmente de forma colectiva. Así, desde nuestro ejercicio nos preguntamos por algunas cuestiones, entre ellas: ¿Cómo propiciar la construcción de conocimiento?; ¿¿ómo transformar las prácticas educativas de la enseñanza de las ciencias?; ¿̇e qué manera se relacionan de forma significativa las temáticas abordadas con el contexto?; ¿qué estrategias favorecen la construcción colectiva de conocimiento?

Una transformación de las prácticas de enseñanza de las ciencias debe contribuir a responder las inquietudes planteadas y, de esta manera, fortalecer las relaciones entre la ciencia y la construcción de conocimiento. En tal sentido, es pertinente recordar que la comunidad científica insiste en la necesidad de no aplazar más las acciones concretas para educar, a las futuras generaciones, en las prácticas para el desarrollo sostenible de nuestra especie en el planeta.

El proyecto disciplinar escolar se considera eje primordial de la actividad en el colegio para construir conciencia científica, da una oportunidad de cambiar la visión cómoda de ver la biología solo como una rama de la ciencia y brinda la ocasión de ir cambiando modelos tradicionales, invitando a los estudiantes a solicitar espacios y tiempos para trabajar en la búsqueda de soluciones ambientales para la institución y el entorno escolar, procurando el desarrollo de valores que garanticen el bienestar y la posibilidad de sobrevivir como especie. De esta manera, se busca incidir directamente en las actuales prácticas ambientales de cada integrante de la comunidad educativa, a través de estrategias pedagógicas y formativas que propendan por la mejora del entorno inmediato y lejano de la institución. 
TEMA: : IMPORTANCIA DE LA INVESTIGACIÓN DE LOS MAESTROS Y MAESTRAS

\section{Referencias}

Elton, C. S. (1927). Animal ecology. Nueva York: Macmillan.

Pozo, M., y Gómez, M. (1990). Aprender y enseñar ciencia. Del conocimiento cotidiano al conocimiento científico. Madrid: Morata.

Reyes, R. (2019). Nichos agroecológicos: Una propuesta para formar líderes ambientales. Semilleros escolares de investigación: memorias de un proceso. Bogotá: SED-IDEP.

Rimbaud, A. (1871). Cartas del vidente: "Yo soy otro" y el "desarreglo de todos los sentidos". Obtenido desde https://www. biblioteca.org.ar/libros/153514.pdf

Rodríguez, M. (2011). La teoría del aprendizaje significativo: una revisión aplicable a la escuela actual. Revista Electrònica d'Investigació i Innovació Educativa i Socioeducativa, Vol. 3, No. 1, pp. 29-50. Obtenido desde http://www.in.uib.cat/ pags/volumenes/vol3_num1/rodriguez/index.html.

Vasco. (1993). Profesionalización docente: conocimiento profesional de los docentes. Trabajo presentado en el III Congreso Internacional de Nuevas Tendencias en la Formación Permanente del Profesorado, Septiembre, Barcelona.

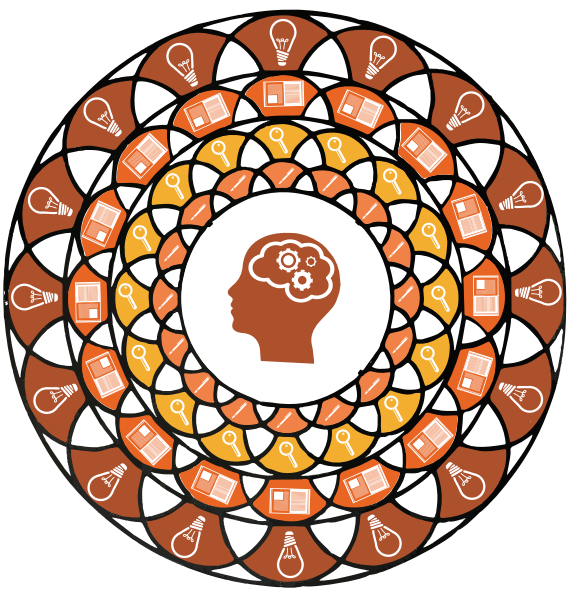

